\title{
Delineating urban functional regions by considering interaction cohesiveness and function diversity
}

\author{
Fei-Ying Kuo $^{\text {a, }}$, Tzai-Hung Wen ${ }^{\text {a }}$ \\ ${ }^{a}$ Department of Geography, National Taiwan University, Fei-Ying Kuo, fykuofelix@gmail.com, Tzai-Hung Wen, \\ wenthung@gmail.com \\ * Corresponding author
}

Keywords: functional region, interaction cohesiveness, function diversity, map equation, urban regionalization

\begin{abstract}
:
A functional region is usually defined as a region characterized by not only interaction cohesiveness but also function diversity. The former indicates that places within a region should share a cohesive interacting pattern of human movements, such as commute or daily travel; the latter means that the places should possess diverse functions like residence, commerce, etc. Due to these two characteristics, people living and moving in one region can acquire almost all their daily needs, and they do not need to frequently move to other regions. In other words, a functional region resembles a life circle. Based on this definition, many previous studies have developed different methods to analyse human flow data so that they can properly profile different types of functional regions like Local labour market area (LLMA) or daily urban system (DUS), which helps people better understand how functional regions compose an area. Existing methods have considered interaction cohesiveness in a functional region, but function diversity is ignored. This is because these studies only used specific-purpose-trip data, such as journey to work or to shop. These kinds of trips describe people moving from their home to their office or to shopping malls, so each trip has an inherent distinction of functions between its origin and destination; thus, a functional region consisting of these trips indirectly owns function diversity. However, this framework has some problems. First, it cannot profile a functional region shaped by composite functions, for only one trip purpose can be taken into account each time. Second, collecting the data of specific-purpose trips needs either some questionnaires or some surveys, so it is often costly and time consuming. Finally, the purpose-unrecorded-trip data provided by rapidly developed smart card systems in several countries can be collected quickly, yet it cannot properly profile functional regions through the aforementioned framework due to the lack of trip purposes. Therefore, we propose a new spatial partitioning algorithm which can simultaneously consider both interaction cohesiveness and function diversity in profiling functional regions. Compared with previous methods, the proposed algorithm can better profile functional regions from many purpose-unrecorded-trip data based on the inclusion of land-use data. Our results provide deeper insights for understanding how various functions of lands and human movements together shape the boundary of a region. It could be significant reference for developing policies for urban planning or public transportation management.
\end{abstract}

